\title{
¿En qué contexto surge la certificación forestal en Chile?: desempeño ambiental, social y económico de empresas no certificadas
}

\author{
In what context did forest certification in Chile arise? Environmental, \\ social and economic performance of non-certified firms
}

\author{
Marcos Tricallotis \\ The Australian National University, Fenner School of Environment and Society, Canberra ACT, Australia, \\ tel.: 61-2-6125 0017, marcos.tricallotis@anu.edu.au
}

\begin{abstract}
SUMMARY
Worldwide, the forest industry faces sustainability challenges that have not been adequately addressed by traditional governance instruments. It is necessary to deepen understanding, from a social sciences standpoint, of the sustainability issues faced by the Chilean forest sector and how - within that context - forest certification arose and evolved. The empirical research reported in this paper sampled 11 plantation forestry and 8 native forestry businesses, and conducted semi-structured interviews of 72 forest industry and forest governance stakeholders in 2013-2014. Results indicated that large plantation forestry businesses, whilst the most economically successful, experienced the largest gaps in environmental and social sustainability - given their covered area. In contrast, native forest owners and small and medium-sized plantation forestry businesses had fewer environmental and social issues, but they were also less economically successful. Due to the perception of international markets and some key stakeholders that traditional state governance was not adequately addressing these sustainability issues, FSC certification arose as an alternative governance mechanism. FSC certification was initially strongly resisted by the large-scale plantation forestry industry, which, with the assistance of the Chilean government, developed a competing national certification scheme: CERTFOR. More recently, major plantation forestry businesses have also adopted FSC certification. It is evident from the research that regulatory changes in plantation forestry, to set clear and equitable rules for all the actors, would enhance achievement of environmental and social sustainability objectives; and that a more appropriate incentive program is necessary to encourage the sustainable management of native forests.
\end{abstract}

Key words: forest governance, forest certification, plantation forests, native forests, and sustainability.

\section{RESUMEN}

Globalmente la industria forestal enfrenta desafíos de sustentabilidad no apropiadamente resueltos por los instrumentos de gobernanza tradicional. Se profundizó, desde las ciencias sociales, en los problemas de sustentabilidad del sector forestal chileno y como en este contexto surgió y evolucionó la certificación de manejo forestal sustentable. Para lograrlo, esta investigación empírica muestreó 11 empresas de plantaciones y ocho empresas de bosque nativo. De esta manera, usando un cuestionario semi-estructurado, se entrevistaron a 72 personas entre 2013 y 2014, provenientes tanto de la industria como de diversos actores claves en gobernanza forestal. Los resultados indicaron que, debido a su mayor extensión, las grandes plantaciones forestales tenían las mayores brechas ambientales y sociales. En cambio, el sector de bosque nativo y pequeños y medianos propietarios de plantaciones tenían menores brechas en sustentabilidad ambiental y social, pero eran mucho menos exitosos económicamente. En este escenario, ciertos mercados internacionales y algunos actores claves en gobernanza forestal percibieron la incapacidad de la gobernanza tradicional estatal para solucionar estas brechas. Consecuentemente, la certificación forestal FSC (Forest Stewardship Council) surgió como un mecanismo de gobernanza alternativo. El FSC fue inicialmente fuertemente resistido por la industria forestal, quienes con ayuda del estado chileno, desarrollaron un esquema alternativo: CERTFOR. Son necesarios, en el caso de las plantaciones, cambios regulatorios para establecer reglas claras y equitativas para todos los actores, que mejoren el desempeño ambiental y social del sector. Similarmente, es aconsejable establecer incentivos más atractivos económicamente para manejar sustentablemente el bosque nativo.

Palabras clave: gobernanza forestal, certificación forestal, plantaciones, bosque nativo, sustentabilidad.

\section{INTRODUCCIÓN}

Es ampliamente conocido que la economía chilena basa su estrategia de desarrollo en exportaciones de materias primas y políticas neoliberales. La industria forestal chilena, en particular, ha sido frecuentemente asociada a externalidades ambientales y sociales negativas en las regiones en donde esta se emplaza (Tricallotis y Kanowski 2016).

A nivel mundial la situación es similar: la deforestación y prácticas deficientes de manejo forestal serían usualmente causantes de diversas externalidades negativas de carác- 
ter ambiental y social. Por ejemplo, la pérdida de bosques nativos impactaría negativamente en comunidades locales y sus servicios ambientales asociados, tales como evitar la emisión de gases de efecto invernadero y control de la erosión e inundaciones (de Groot y van der Meer 2010).

En Chile hay dos sectores forestales: un sector industrial basado en plantaciones exóticas de diferentes especies de pinos (principalmente de Pinus radiata D. Don) y eucaliptos (del género Eucalyptus); y un sector dependiente del bosque nativo (donde predominan especies del género Nothofagus). Cabe señalar que los productos forestales son obtenidos exclusivamente, en ambos sectores forestales, por empresas privadas (Leyton 2009).

Económicamente, las plantaciones forestales contribuyen importantemente al producto interno bruto (PIB) chileno (US\$ 4.829 millones), siendo el tercer sector exportador (INFOR 2015). Cabe señalar que el establecimiento de las plantaciones a gran escala fue promovido durante la dictadura militar de Augusto Pinochet, bajo el decreto ley (DL) 701 de 1974. Inicialmente, el objetivo fue promover la forestación de suelos agrícolas degradados en regiones del centro y sur del país, lo cual permitió un crecimiento exponencial de las plantaciones: desde alrededor de 300.000 hectáreas en 1975 se alcanzaron 2,4 millones de hectáreas en 2013 (INFOR 2015). Esta superficie se concentra (aproximadamente un $63 \%$ ) en grandes empresas chilenas (sobre 50.000 hectáreas según DL 701), las que también poseen la tierra (no existen concesiones forestales en Chile, ni para plantaciones ni para bosque nativo), y que exportan aproximadamente US\$ 5.439,1 millones a países de Norte América, Asia, Europa, Latino América y, crecientemente, a China (INFOR 2016). Hoy las dos mayores empresas chilenas se han transformado en multinacionales, operando principalmente en Latinoamérica.

La industria de plantaciones produce aproximadamente 42,6 millones de metros cúbicos al año de madera en rollizos; de estos se obtiene pulpa y astillas mayoritariamente para exportación, y madera aserrada para ser consumida principalmente en el mercado doméstico (INFOR 2015).

En cambio, el bosque nativo no provee el mismo valor económico que las plantaciones pero cubre 13,4 millones de hectáreas, de las cuales alrededor de cuatro millones pertenecen y son explotadas principalmente por pequeños y medianos propietarios (menor a 50.000 hectáreas según DL 701, si bien propiedades de 12 hectáreas son usuales para pequeños propietarios) (Leyton 2009). El principal producto que se obtiene del bosque nativo es la leña, orientada principalmente al mercado doméstico (Bustamante y Díaz 2010); si bien existe cierta proporción de madera aserrada (valorada en US\$ 4,9 millones), principalmente de lenga (Nothofagus pumilio Poepp et Endl), que se exporta a países latinoamericanos, asiáticos y Estados Unidos (INFOR 2016). Aunque en 2008 se promulgaron regulaciones específicas para dinamizar económicamente el sector (Ley No 20823 y reglamentos), estas no habrían surtido el efecto esperado según estas cifras.
Institucionalmente, existen dos agencias relevantes: la Corporación Nacional Forestal (CONAF) y el Instituto Forestal (INFOR). CONAF está encargada de supervisar el cumplimiento de la legislación forestal y evitar la deforestación (que consiste en cortar un "bosque", según criterios establecidos en Ley No 20823; ejemplo: superficie mínima de $5.000 \mathrm{~m}^{2}$ y predominio de árboles de $5 \mathrm{~m}$ de altura); también otorga los derechos de explotación para plantaciones (aquellas acogidas a subsidios según DL 701) y bosque nativo (solo para algunas especies, de los géneros Nothofagus, Quillaja, Laureliopsis y Podocarpus spp., por nombrar algunas), previa aprobación de un plan de manejo forestal elaborado por un profesional forestal. Usualmente, este plan contiene los requisitos detallados de manejo sustentable, incluyendo: métodos y diámetros de corta; medidas de regeneración forestal y protección del suelo/ agua; y, tratamiento de los desechos, entre otros. Este plan debe cumplir con normas de buenas prácticas CONAF y regulaciones específicas (cuadro 1).

INFOR provee asistencia técnica para apoyar la toma de decisiones en el sector forestal. Adicionalmente, aunque la Dirección del Trabajo no es una agencia forestal, supervisa el cumplimiento de las regulaciones laborales en este sector. En el cuadro 1 se presentan algunos de los principales hitos en política forestal chilena, apreciándose que las regulaciones particulares han más bien "moldeado" la política forestal y que solo desde 2016 existe una política explícita.

Globalmente, ambos tipos de industrias tienen diferentes desafíos para su sustentabilidad que no han sido apropiadamente resueltos por los instrumentos de gobernanza forestal estatal tradicional. Gobernanza se refiere a quien o quienes detentan la autoridad para dictar y hacer cumplir determinadas normas o instrumentos de política forestal para alcanzar ciertos objetivos colectivos (por ejemplo, promover prácticas forestales sustentables y a la vez bienestar económico local); usualmente, son los estados-nación quienes detentan esa autoridad soberana dentro de un determinado territorio (Auld 2014). No obstante, las regulaciones estatales tradicionales suelen ser insuficientes per se para evitar problemas ambientales, sociales y económicos, dados sus mecanismos para asegurar su cumplimiento (Gunningham 2009).

En Chile, los problemas ambientales asociados a las plantaciones pueden resumirse en cierta substitución de bosque nativo por estas así como el impacto de sus operaciones en suelos y agua (Nahuelhual et al. 2012, Reyes y Nelson 2014). El sector bosque nativo, en cambio, sufriría de tala ilegal y degradación por décadas de "floreo" o cosecha selectiva no-sustentable (Lara et al. 2013), la cual consiste en extraer los mejores árboles y dejar los peores ejemplares. En materia social, hay dos realidades. Si bien en el sector bosque nativo chileno no hay demasiados estudios, Kausel y Vergara (2003) constatan una relación positiva entre emprendimientos forestales y comunidades locales (destacando las donaciones de leña hacia estas últimas); en contraste, este sector presentaría frecuentes deficiencias en 
Cuadro 1. Principales hitos en política forestal chilena.

Main milestones in Chilean forest policy.

\begin{tabular}{ccl}
\hline Hito & Período/Año & \multicolumn{1}{c}{ Descripción } \\
\hline Ley N 4363 & 1931 - presente & $\begin{array}{l}\text { La ley, que ha sufrido sucesivas modificaciones, definió los suelos de aptitud } \\
\text { forestal, restricciones al uso del fuego, prohibición de cortar árboles y arbustos } \\
\text { necreto No } 2374 \\
\text { de agua y en pendientes sobre } 45 \% \text {. El reglamento estableció restricciones } \\
\text { para cortar bosques cercanos a cursos de agua, particularmente sobre "excesi- } \\
\text { vas pendientes". }\end{array}$ \\
\hline
\end{tabular}

Decreto Ley (DL) 701 - $\quad 1974-2012$

Ley de Desarrollo Forestal
Esta ley marca el hito más importante para desarrollar el sector forestal: promovió la participación de actores privados, mediante subsidios (75\% de los costos) para forestar masivamente suelos agrícolas degradados con plantaciones exóticas de pino y eucalipto. La ley expiró en 2012; sin embargo, durante los últimos cuatro años parte del parlamento chileno y dos gobiernos en ejercicio han discutido extenderla, aún sin éxito.

\begin{tabular}{lcl}
\hline $\begin{array}{l}\text { Privatización de la industria } \\
\text { forestal }\end{array}$ & $1974-1976$ & $\begin{array}{l}\text { El estado transfirió sus empresas forestales al sector privado, en un proceso } \\
\text { altamente cuestionado. }\end{array}$ \\
\hline Decreto No 259 & 1980 - presente & $\begin{array}{l}\text { Este reglamento especifica la operación del DL 701, estableciendo requer- } \\
\text { imientos generales para el manejo forestal. }\end{array}$ \\
\hline Decreto No 193 & 1998 - presente & $\begin{array}{l}\text { Este reglamento especifica la operación del DL 701, actualizando el reglamen- } \\
\text { to N } 259 \text { de 1980. También establece que suelos son "forestables" (por ejem- } \\
\text { plo, suelos degradados y en proceso de desertificación) y hace obligatoria los } \\
\text { planes de manejo forestal en tales suelos. }\end{array}$ \\
\hline
\end{tabular}

Ley No 20283 - Ley de Recuperación de Bosques Nativos y Desarrollo Forestal (Ley de Bosque Nativo)

Reglamento de suelos, aguas 2011 - presente y humedales (DS No 82)

Promulgación Política

Forestal en 2016

$2015-2035$
2008 - presente

Esta ley establece incentivos mediante subsidios (que pueden ir entre US\$350 a $\$ 700$ por hectárea, por mes, y dependiendo que actividad se realice) a bosques sustentablemente manejados. Sin embargo, la ley no habría tenido los resultados esperados (Lara et al. 2013).
Este reglamento establece los requerimientos específicos que los propietarios de bosque nativo deben seguir para proteger zonas ribereñas (particularmente, áreas de alto valor de conservación), especificando los anchos desde los bordes de los cursos de agua temporales/permanentes, cuerpos de agua y quebradas.

Este documento establece los pilares para un desarrollo forestal sustentable: (a) institucionalidad forestal, (b) productividad y crecimiento económico, (c) inclusión y equidad social, y (d) protección y restauración del patrimonio forestal.

Fuente: elaboración propia basada en Reyes y Nelson (2014).

aspectos de salud y seguridad ocupacional (Otero 2006). En el caso de las plantaciones, a pesar de que las condiciones en materia laboral serían mejores, persistirían importantes problemas en la relación de empresas grandes con trabajadores forestales (principalmente subcontratados), pequeñas empresas forestales, y contratistas de servicios (OIT Santiago 2012, Meyer y Tappin 2014, Tricallotis y Kanowski 2016), así como serios conflictos con comunidades indígenas por la tenencia de la tierra (Gerber 2011, Reyes y Nelson 2014, Andersson et al. 2016). Económicamente, las plantaciones forestales chilenas serían exitosas; en contraste, el sector bosque nativo ha tenido que luchar con bosques degradados y de baja calidad, dificultades en la cadena de comercialización, bajos precios, y estrechas utilidades (Bustamante y Díaz 2010). De todas estas externalidades negativas mencionadas, la gobernanza forestal estatal habría sido incapaz de resolverlas.

Auld (2014) plantea que, debido a las limitaciones de la gobernanza estatal tradicional, los enfoques de "gobernanza forestal no-estatal" surgen como alternativas a la regulación tradicional. En particular, la certificación de manejo forestal sustentable es promovida como respuesta a los problemas de sustentabilidad de la industria por diversos actores, incluyendo organizaciones no gubernamentales (ONG), sectores de la sociedad civil, gobiernos $\mathrm{y}$ asociaciones industriales. Ciertamente, a pesar de que existen diferencias entre las aspiraciones de actores tan diversos (tales como la industria y ciertas ONG locales 
e internacionales), existe una visión común en mejorar la sustentabilidad de la industria forestal en su conjunto.

La hipótesis de investigación plantea que los instrumentos de gobernanza estatal tradicional no han sido capaces de responder a los desafíos ambientales, sociales y económicos del sector forestal chileno. Teniendo en cuenta lo anterior, los objetivos de este trabajo son: a) identificar los aspectos históricos, políticos, ambientales, sociales y económicos del sector forestal que han determinado el éxito o fracaso de la gobernanza forestal tradicional; $b$ ) evaluar las externalidades del sector forestal, mediante las ciencias sociales, desde las visiones, experiencias y percepciones de los diferentes actores en gobernanza forestal; y c) conocer cómo, cuándo y porqué, dado el contexto anterior, surge la certificación forestal como una alternativa a la gobernanza estatal. Este trabajo, metodológicamente, profundiza en los objetivos anteriores al obtener descripciones detalladas desde los estudios de caso que aquí se exponen.

\section{MÉTODOS}

Para realizar esta investigación se utilizó principalmente el marco metodológico de Tikina e Innes (2008) para medir cualitativamente la efectividad de la certificación forestal, en términos de: (a) resolver problemas de sustentabilidad; (b) alcanzar ciertas metas (ejemplo: acceso de empresas a mercados/licencia social para operar); (c) adoptar estándares de certificación (efectividad del proceso); (d) aceptación por la sociedad (efectividad constitutiva); y (e) eficiencia, equidad y robustez (efectividad evaluativa). Con esta metodología se buscó más bien responder a la hipótesis de investigación, esto es, la incapacidad de los instrumentos de gobernanza estatal en términos de resolver problemas (a) y alcanzar metas (b) de sustentabilidad. Es decir, se investigó acerca del contexto en aspectos políticos, ambientales, sociales y económicos de empresas sin certificación, o su situación previo a obtenerla, y como estas condiciones habrían propiciado la adopción de la certificación.

Los sitios de estudio fueron aquellas regiones del país donde la actividad forestal era más relevante, específica- mente las regiones del Maule, Biobío, La Araucanía, Los Ríos, Los Lagos y Magallanes. De estas regiones, se muestrearon 19 empresas forestales de bosque nativo y plantaciones, mediante selección de casos diversos y muestreo dirigido (Neuman 2011), siendo los criterios empleados: variedad/tipo de especies forestales; cantidad de hectáreas; orientación de mercado (doméstico o internacional); nivel de sofisticación tecnológica, material y en recursos humanos; y presencia/ausencia de conflictos ambientales y sociales. Así se obtuvo una muestra formada por empresas pequeñas, medianas y grandes, de acuerdo a la clasificación del FSC (Forest Stewardship Council) (cuadro 2). De esta manera, si bien no se obtuvo una muestra representativa en términos estadísticos, esta fue diversa, permitiendo mejorar el entendimiento del fenómeno estudiado desde un escenario real. Sin embargo, dos grandes empresas de plantaciones muestreadas abarcaban la mayor superficie plantada (sobre $50 \%$ ) del país; similarmente, dos empresas de bosque nativo muestreadas eran las más importantes en términos de sofisticación tecnológica, material, recursos humanos y cantidad de hectáreas.

Se emplearon métodos cualitativos para recolectar y analizar los datos, entrevistándose a 72 personas entre 2013 y 2014; para lograr esto, se utilizó un cuestionario semi-estructurado siguiendo un protocolo de ética para respetar la confidencialidad y derechos de los informantes (ANU Human Ethics Protocol No 2012/250). La muestra de entrevistados incluyó no solo administradores y propietarios forestales, sino que también diversas partes interesadas (cuadro 3) para obtener la mayor heterogeneidad de visiones posibles, usando diferentes métodos no probabilísticos: en particular, muestreo dirigido, secuencial, desviante y de redes (Neuman 2011).

El cuestionario incluyó preguntas solicitando información acerca de los desafíos ambientales, sociales y económicos experimentados por el sector durante los últimos 20 años hasta 2013-2014, considerando el contexto histórico y político. Se utilizó una estrategia múltiple para triangular los datos de las entrevistas (Layder 1998): esto consistió en contrastar la información primaria de las entrevistas con información secundaria obtenida desde diversas fuentes incluyendo documentos de gobierno (ejemplo:

Cuadro 2. Clasificación del tamaño de las empresas según las hectáreas de la unidad de manejo forestal.

Business scale as number of hectares of the forest management unit (FMU).

\begin{tabular}{lll}
\hline \multicolumn{1}{c}{ Categoría } & Superficie de la unidad de manejo forestal & Empresas seleccionadas en la muestra \\
\hline Pequeña escala & $\leq 1.000$ hectáreas & 3 de bosque nativo y 5 de plantaciones \\
Mediana escala & Entre pequeña y mediana escala & $5^{*}$ de bosque nativo y 4 de plantaciones \\
Gran escala & $>80.000$ hectáreas (plantaciones) & 2 de plantaciones \\
& $>300.000$ hectáreas (bosques naturales) & \\
\hline
\end{tabular}

Fuente: FSC Guía de escala, intensidad y riesgo (EIR) para los encargados del desarrollo de estándares (FSC-GUI-60-002 V1-0 ES).

* De estas cinco empresas medianas, dos tenían niveles de sofisticación de empresas relativamente grandes. 
informes sectoriales, leyes, reglamentos y guías de manejo forestal), estadísticas e informes públicos (ejemplo: estadísticas laborales de organismos públicos e informes de pre-certificación de casas certificadoras de las empresas muestreadas), e información de prensa. Posteriormente, las entrevistas fueron transcritas y luego codificadas mediante el programa computacional QSR NVIVO. Los datos se analizaron usando redes temáticas (Attride-Stirling 2001), las cuales los organizan buscando premisas evidentes en estos hasta llegar a altos niveles de abstracción. Adicionalmente, se utilizó el método comparativo (Hopkin 2010), para verificar si existían patrones comunes entre los diferentes estudios de caso investigados; por ejemplo, cómo influenciaba la ausencia de certificación tanto a empresas de plantación como a las de bosque nativo.

\section{RESULTADOS}

\section{PLANTACIONES FORESTALES}

El exitoso establecimiento de las plantaciones forestales en Chile durante la década de 1970 ha sido cuestionado por ciertos actores. Según varios representantes indígenas y miembros de ONG este proceso se realizó de manera ilegítima e irregular. Primero, los cambios en la política forestal fueron hechos en ausencia de un debate público,

Cuadro 3. Resumen de entrevistados por categoría.

Summary of interviewees by category.

\begin{tabular}{ll}
\hline \multicolumn{1}{c}{ Tipo de entrevistado } & $\begin{array}{c}\text { Número de } \\
\text { entrevistados }\end{array}$ \\
\hline $\begin{array}{l}\text { Plantaciones: administradores y } \\
\text { propietarios forestales }\end{array}$ & 18 \\
$\begin{array}{l}\text { Bosque nativo: administradores y } \\
\text { propietarios forestales }\end{array}$ & 10 \\
Asociaciones forestales & 3 \\
Contratistas forestales & 4 \\
Trabajadores forestales & 2 \\
Representantes sindicales & 4 \\
Miembros de comunidades no indígenas & 2 \\
Miembros de comunidades indígenas & 4 \\
Representantes de ONG nacionales & 7 \\
Autoridades forestales & 8 \\
Autoridades laborales & 2 \\
Investigadores y consultores forestales & 5 \\
Representantes de FSC Chile & 1 \\
Representantes de CERTFOR Chile & 2 \\
\hline
\end{tabular}

sin oposición política y, en consecuencia, las nuevas leyes carecerían de consentimiento democrático. Segundo, este proceso permitió privatizar cuantiosos recursos forestales y tierra, concentrando la propiedad forestal en pocos propietarios, quienes amasaron inmensas fortunas y monopolizaron la cadena de abastecimiento. Tercero, el establecimiento de las plantaciones habría sido a expensas de pequeños campesinos y pueblos indígenas, quienes fueron forzados a vender sus tierras a bajo precio con el beneplácito de las autoridades militares. Según lo señalado por este representante indígena mapuche:

"Dentro de estas corporaciones había gente muy poderosa...incluso gente vinculada al gobierno militar, había generales que estaban involucrados (...) la violencia era muy común en ese tiempo y porque a la gente se le intimidaba, terminaba vendiendo sus tierras a 'precios de ganga'. Y ellos [las corporaciones] ganaron billones comprando así." (entrevista con I-IX-01).

No obstante, algunos investigadores y asociaciones forestales reconocieron ciertos impactos ambientales positivos del establecimiento de plantaciones: la recuperación de suelos agrícolas erosionados. Ellos concordaron que la expansión de las plantaciones fue parte de un esfuerzo estatal para detener la erosión en la zona centro sur, situación crítica para el país en el siglo XX.

Desafortunadamente, la expansión de las plantaciones también fue a expensas de bosques nativos, según investigadores y miembros de la misma industria forestal reconocieron. Existe, sin embargo, controversia acerca del monto de tal sustitución. Mientras algunas estimaciones por parte de la industria reconocen no más de 33.000 hectáreas substituidas, otros reportes (OIT Santiago 2012) estiman sobre 200.000 hectáreas de bosques nativos substituidos en la zona sur y centro sur del país entre 1974 y 1992.

En términos organizacionales, las mayores empresas forestales del país poseen el más vasto patrimonio forestal y controlan la cadena de abastecimiento maderero (incluyendo el procesamiento, transporte y comercialización). De esta forma, pueden reducir costos y aumentar la eficiencia de sus operaciones, altamente mecanizadas y subcontratadas. Esta estrategia fue cuestionada durante las entrevistas por miembros de comunidades locales, representantes sindicales, contratistas y ciertas ONG, debido a que iría en desmedro de contratistas y pequeños propietarios forestales. Por ejemplo, algunos de estos entrevistados señalaron que esta agresiva estrategia obligaría a los contratistas forestales a mantener tarifas muy bajas y a sufrir el retraso de varios meses por el pago de sus servicios.

Por otra parte, los resultados sugieren que a diferencia de las pequeñas y medianas empresas, las grandes corporaciones forestales eran en general altamente visibles y blanco frecuente de críticas por parte de activistas ambientales y organizaciones sociales, asociando su actuar a numerosos problemas sociales y ambientales. 
Desempeño ambiental. En general, debido a contar con mayores recursos, las grandes corporaciones forestales han podido usar más intensivamente tecnologías de menor impacto ambiental que empresas más pequeñas (cuadro 4). Por ejemplo, según fuentes de la industria, estas empresas cosechaban mediante métodos mecanizados haciendo uso de maquinaria feller-bunchers, harvesters y torres de madereo en terrenos planos y pendientes (bajo 35 grados) la mayoría de las veces. Todos estos métodos de cosecha, responderían de acuerdo a representantes sindicales y de la industria, a una estrategia de reducción de costos y no, inicialmente al menos, a motivaciones ambientales.

No obstante, la mayor extensión de las operaciones forestales de grandes plantaciones magnificaría también su impacto ambiental negativo. El más importante sería la extensión de las talas rasas. A este respecto, varios entrevistados sugirieron que las grandes empresas de plantaciones tenían pocas restricciones: sus talas rasas frecuentemente alcanzaban extensiones sobre 400 hectáreas anuales.

Por otra parte, las operaciones forestales ejecutadas por pequeños y medianos propietarios (cuadro 4) harían uso de diferentes métodos de cosecha: desde el método tradicional, usando bueyes y motosierras, hasta métodos algo más sofisticados, utilizando maquinaria como skidders y cargadores tri-neumáticos (mayormente en medianas empresas).

En términos generales, la muestra analizada de pequeñas y medianas empresas forestales mostró un disímil desempeño ambiental: mientras existiría una mayoría de propietarios que no cumpliría la ley y prácticas forestales sustentables, una minoría sí lo haría (cuadro 4). En relación al primer grupo, un contratista forestal describió sus prácticas:

"Bueno, los particulares dejan lo mínimo [de zonas ribereñas] porque nadie controla aquí. Ellos están solamen- te interesados en sacar lo máximo, ellos no dejan los 10 o 20 metros que se requiere [de zona ribereña]. No hay control de CONAF, o de nadie...ellos [pequeños propietarios] se instalan en cualquier lado y solo cortan. Ellos no están preocupados de eso [de cumplir las leyes forestales]." (entrevista con CT-VIII-t01).

Otros ejemplos de prácticas forestales deficientes, identificadas en este estudio, incluyeron: quemas habituales de residuos forestales, desarrollo de operaciones de cosecha en épocas lluviosas (ejecutadas sin consideraciones ambientales) y contaminación del suelo y cursos de agua con residuos peligrosos (por aceites y lubricantes de maquinaria forestal). En la opinión de varios entrevistados, muchos pequeños propietarios forestales operarían con relativa impunidad debido a la baja probabilidad de ser fiscalizados por la autoridad forestal (CONAF).

En relación al segundo grupo de pequeños y medianos propietarios, si bien falta mayor evidencia, ciertos propietarios de empresas agroforestales cumplirían la ley y seguirían prácticas forestales sustentables para no perjudicar la parte agrícola de su negocio. Por ejemplo, las quemas de residuos y la aplicación de productos químicos estarían estrictamente prohibidas.

Desempeño social. Los resultados sugieren que el desempeño en materia social de las empresas, en relación con sus trabajadores forestales y comunidades locales, variaba de acuerdo al tamaño de estas. En primer lugar, en relación con los trabajadores forestales, la mayoría de los entrevistados concordó que en el caso de grandes empresas forestales, las condiciones laborales (relativo a campamentos forestales, alimentación y transporte de trabajadores) y de seguridad ocupacional habrían mejorado significativamen-

Cuadro 4. Desempeño y principales problemas de sustentabilidad de la industria de plantaciones forestales.

Main sustainability issues and performance of the plantation forest industry.

\begin{tabular}{lll}
\hline Desempeño ambiental & - & Sustitución de bosque nativo por plantaciones. \\
& $\bullet$ & Impactos negativos de (extensas) talas rasas en suelo y cursos de agua. \\
& - & Polución causada por productos químicos (ejemplo: fumigaciones aéreas). \\
\hline Desempeño social & - & Trabajadores forestales: pobres condiciones laborales y de salud y seguridad ocupacional (algunas \\
& empresas contratistas y pequeños propietarios), sobrecarga de trabajo, prácticas anti-sindicales \\
& (contratistas de grandes empresas) y deficiente capacitación. \\
& Comunidades locales: conflictos con comunidades indígenas por la tenencia de la tierra (grandes \\
& empresas), escasos beneficios y molestia para las comunidades locales derivados de las operacio- \\
& nes forestales (grandes empresas). \\
\hline Desempeño económico & Grandes empresas: sector exportador económicamente exitoso. \\
- Pequeñas y medianas empresas: menores beneficios debido al monopolio ejercido por grandes \\
empresas.
\end{tabular}

Fuente: entrevistas del autor e información secundaria. 
te en los últimos quince años. Este progreso, según investigadores forestales, se debería a la presión ejercida por organizaciones sindicales. Adicionalmente, el progreso de la legislación laboral chilena también habría contribuido a mejorar las condiciones laborales (en particular la ley 20123 de 2006, que reguló la relación con subcontratistas), según el testimonio de inspectores laborales entrevistados.

Sin embargo, la progresiva mecanización de las operaciones forestales requeriría menos trabajadores pero de mayor calificación. Más aún, de acuerdo a fuentes de la industria, las empresas enfrentaban escasez de mano de obra, debido a menores beneficios sociales y remuneraciones que esta industria ofrecería en comparación con otras, como la minería. Consecuentemente, los trabajadores forestales sufrirían mayores presiones por hacer horas extra y no tomar vacaciones. Y a su vez, mostrarían signos de fatiga temprana, baja productividad, y problemas familiares debido a largos períodos en campamentos forestales. Según lo describieron algunos representantes sindicales:

\footnotetext{
"En el equipo que yo trabajo hay 26 trabajadores; mis compañeros y yo nos levantamos a las 5.30 am y llegamos a la casa a las $7.00 \mathrm{pm}$ para ganar más monedas, incluso con las tremendas calores. Mis compañeros que trabajan con la motosierra tienen una pausa a las 10.00 am y luego a las $12.00 \mathrm{pm}$. A las $02.00 \mathrm{pm}$ hay otra pausa, pero aparte de eso la motosierra no para de sonar todo el día. Nosotros solo queremos trabajar lo normal y no matarnos trabajando para hacer un poco más.” (entrevista con IW-MB-03).
}

Más aún, se detectaron debilidades en el programa de calificación laboral administrado por CORMA (Corporación Chilena de la Madera, asociación que agrupa principalmente a grandes empresas); el programa, según representantes sindicales e investigadores forestales, fallaría en la preparación teórica y práctica de los trabajadores forestales. Según un investigador forestal:

"Un día que el operador de skidder estaba ausente, ellos [los trabajadores] le pasaron esta máquina a un tipo que no tenía idea de cómo manejarla y él se demoraba cinco veces más que un operador ya formado. Hay muchas cosas que se aprenden en la marcha, pero sin comprender el fundamento técnico. No sé cómo no más gente ha muerto. Hay mucha improvisación.” (entrevista con R-MB-03).

También se identificó una alta variación en las condiciones laborales entre empresas contratistas forestales. $\mathrm{Si}$ bien varias tenían condiciones laborales apropiadas, otras tenían condiciones precarias según lo expuesto por varios entrevistados; esto se debería a que habría contratistas en mejor condición financiera para reducir el precio de sus servicios a solicitud de empresas forestales. Más aún, representantes sindicales denunciaron la existencia de prácticas anti-sindicales en varias empresas contratistas, incluyendo: despidos injustificados, cláusulas que prohibían huelgas en contratos de prestación de servicios entre contratistas y empresas forestales, persecución contra dirigentes, obstrucción a procesos de negociación colectiva y discriminación en contra de trabajadores sindicalizados.

En relación con los trabajadores de pequeñas y medianas empresas forestales, las condiciones laborales eran en general bastante más precarias. La mayoría de los entrevistados reconoció así que las condiciones laborales distaban mucho de los estándares de grandes empresas: se ofrecerían menores salarios, escasos beneficios sociales, baja capacitación y deficiente seguridad ocupacional debido a la falta de elementos de protección personal. En consecuencia, según investigadores forestales e inspectores laborales, el sector sería poco atractivo laboralmente; siendo además mucho más probable encontrar trabajadores envejecidos (escasamente requeridos por grandes empresas) y también trabajadores muy jóvenes o inexpertos.

En segundo lugar, relativo a comunidades locales, la relación de las grandes empresas forestales con estas sería conflictiva. Como se comentó anteriormente, el proceso de privatización y creación de grandes patrimonios forestales durante las décadas de 1970s y 1980s habría exacerbado un conflicto indígena de larga data originado en la guerra de pacificación de La Araucanía a fines del siglo XIX. Según lo señalado por un gerente de la industria:

"Y el resultado final fue que los mapuches fueron forzados a vivir en unas pocas hectáreas [aludiendo a su estilo de vida nómade], cada familia era propietaria de 20 hectáreas. Con suerte cada familia tiene media hectárea hoy, porque la tierra fue dividida en las sucesivas generaciones. Ellos sobreexplotaron sus bosques nativos, no dejando nada que sobreviviera...luego, y esto es bastante obvio; si ellos ya no tenían bosques entonces buscaron árboles de plantaciones y esa es la razón por la que los árboles son robados de nuestros predios." (entrevista con PFB-MB-q01).

El así llamado conflicto Mapuche se habría tornado violento desde fines de los años 1990s. Esta violencia se ha traducido en ataques a camiones forestales, amenazas a personal forestal, tiroteos e incendios intencionales a plantaciones por parte de una minoría de violentos activistas como varios entrevistados de la industria reconocieron. Parte importante de la responsabilidad en este conflicto, según varias ONG y miembros de la misma industria coincidieron, sería debido a que las empresas forestales ignoraron largamente a sus comunidades: al mecanizar sus operaciones (reduciendo fuentes de empleo local), generar molestia y daños (por las operaciones de transporte forestal y fumigación), cercar sus plantaciones (prohibiendo la recolección de productos forestales no maderables [PFNM] y leña sobrante), y no dialogar con las comunidades, generaron resentimiento desde estas hacia la gran industria forestal. 
En contraste, en la mayoría de las entrevistas con propietarios de pequeñas y medianas empresas forestales se destacó la escasez de conflictos con comunidades. Esta información fue consistente con lo manifestado por miembros de ONG, consultores e investigadores forestales. En general, estos emprendimientos y comunidades locales indígenas y no indígenas cultivaban una relación mutualmente colaborativa: las empresas proveían PFNM, leña, y empleos a las comunidades, y estas a su vez cuidaban las plantaciones.

Desempeño económico. Las grandes empresas de plantaciones se han caracterizado por ser un sector económicamente exitoso y orientado hacia las exportaciones. Sin embargo, debido a que han controlado la cadena de abastecimiento, pequeños y medianos propietarios se sentían perjudicados al obtener bajos precios por metro cúbico de madera aserrada. Ellos, y miembros de comunidades locales, criticaron fuertemente este monopolio, percibiéndolo como abusivo.

\section{BOSQUE NATIVO}

Generalmente, las empresas de bosque nativo representan un sector modesto tanto en términos económicos, técnicos y productivos (cuadro 5).

Desempeño ambiental. Si bien una cantidad importante de pequeñas operaciones forestales en Chile siguen prácticas deficientes e ilegales de manejo forestal (Lara et al. 2013), la muestra analizada de empresas (formales) reveló que seguían un plan de manejo forestal aprobado por CONAF, realizando cosecha con métodos de baja intensidad, incluyendo cortas de selección o en fajas. En general, estos propietarios forestales (de las regiones de La Araucanía, Los Ríos y Los Lagos) reconocieron trabajar con métodos de menor impacto ambiental: cosecha cuidadosamente planificada, arrastre de troncos usando bueyes, cosecha restringida en pendientes mayores a 45 grados, exclusión de ganado del bosque para facilitar la regeneración de renovales, planificación y mantenimiento de caminos forestales, y protección de cursos de agua y zonas ribereñas.

Un caso particular es el manejo de los bosques de lenga por empresas medianas en la región de Magallanes, en el extremo sur. Según la mayoría de los entrevistados, desde hace aproximadamente quince años esta especie se manejaba de manera sustentable (ejemplo: métodos de cosecha incluían cortas de protección) y consensuada entre autoridades, universidades y la industria local. Según lo expresado por un administrativo forestal:

\begin{abstract}
"Nosotros tenemos apoyo científico y a CONAF respaldándonos también. CONAF, la Universidad de Chile y esta empresa están trabajando juntos para manejar la lenga en Magallanes. Y qué bueno que fue siempre así. Eso es fundamental y no tendría sentido hacerlo de otra forma. CONAF de hecho aplica esos criterios [de las investigaciones científicas en manejo de lenga] en la interpretación de los planes de manejo y así estos son aprobados." (entrevista con NFB-XII-c01).
\end{abstract}

Sin embargo, el manejo forestal sustentable del bosque nativo suponía varias dificultades debido a décadas de cosecha selectiva no-sustentable o "floreo". Esto, en la opinión de varios propietarios, les dificultaba extraer mayores porcentajes aserrables debido al estado degradado del bosque.

Desempeño social. Las condiciones laborales serían usualmente precarias en pequeñas operaciones forestales de bosque nativo. Según algunos inspectores laborales, muchas de estas empresas eran pequeños emprendimientos que frecuentemente contrataban vecinos y familiares, pero que no "hacían el papeleo" y que también carecían de la formalidad necesaria para proveer condiciones básicas de seguridad ocupacional. Estas condiciones diferían de aquellas brindadas por empresas más grandes, según un inspector laboral:

Cuadro 5. Desempeño y principales problemas de sustentabilidad de la industria de bosque nativo.

Main sustainability issues and performance of the native forest industry.

\begin{tabular}{ll}
\hline Desempeño ambiental & - Cierto grado de polución química causado por la maquinaria de operaciones forestales. \\
& - Tala ilegal y no sustentable (pequeñas operaciones forestales, en empresas informales). \\
\hline Desempeño social & $\begin{array}{l}\text { Trabajadores forestales: condiciones laborales modestas, deficiente capacitación, y pobre desem- } \\
\text { peño en salud y seguridad ocupacional. }\end{array}$ \\
& $\begin{array}{l}\text { - Comunidades locales: ausencia de conflictos con comunidades, más bien relación de mutua cola- } \\
\text { boración entre empresas y comunidades. }\end{array}$ \\
\hline Desempeño económico & $\begin{array}{l}\text { Modesto desempeño económico debido a bajos precios, problemas en la cadena de comercializa- } \\
\text { ción y baja calidad de la madera. }\end{array}$
\end{tabular}

Fuente: entrevistas del autor e información secundaria. 
"Ellos [los trabajadores forestales] tienen buen alojamiento, las comidas son buenas y el transporte a sus hogares desde los campamentos forestales es bueno también." (entrevista con La-XII-01).

Aparentemente, el tamaño de las operaciones forestales influenciaba positivamente las condiciones laborales y de seguridad ocupacional, si bien persistían ciertas falencias en algunas empresas. Otro aspecto importante es la ausencia de prácticas anti-sindicales en empresas medianas. Incluso, ciertos administradores forestales valoraron el rol de los sindicatos en lograr mejoras en las condiciones laborales y productividad para la empresa mediante procesos de negociación colectiva.

En relación con las comunidades locales, ninguna empresa de bosque nativo mantenía conflictos con estas. La relación era positiva en términos generales. Las pequeñas y medianas (región de Magallanes) empresas proveían fundamentalmente de trabajo a comunidades indígenas (etnia Mapuche) y no indígenas, respectivamente. Adicionalmente, ciertas empresas tenían acuerdos con sus comunidades locales para donarles leña, quienes mantenían libres de residuos las canchas de acopio de madera.

Desempeño económico. Comparada con las plantaciones, la industria de bosque nativo sería modesta en términos económicos. Según diversas fuentes entrevistadas, esto obedecería a varias razones. La primera es que los bosques nativos degradados ofrecerían un muy bajo porcentaje de madera aserrable (usualmente no más de un $20 \%$ ) lo que no permitiría obtener mejores precios. Esto haría que gran parte de la producción enfocara en la producción de leña. Segundo, los largos ciclos de crecimiento (mayores de 40 años) de las especies nativas harían difícil obtener retornos económicos relativamente rápidos y atractivos como en el caso de las plantaciones forestales. Tercero, la cadena de comercialización, particularmente para los pequeños propietarios, carecería de la profesionalización y los eslabones necesarios para funcionar apropiadamente. Más aún, los pequeños propietarios forestales acusaban una burocracia estatal injustificada para desarrollar sus operaciones y la vez sentían que la ley de bosque nativo (ley 20823) no había traído los beneficios esperados. No obstante, debido a economías de escala, las empresas de mayor tamaño que explotaban la lenga en Magallanes tenían un mejor desempeño económico relativo que los pequeños propietarios forestales.

\section{SURGIMIENTO Y EVOLUCIÓN DE LA CERTIFICACIÓN FORESTAL}

Bajo el contexto anterior, no fue de extrañar que tanto sectores de la industria como ciertas partes interesadas (ONG nacionales e internacionales principalmente) buscaran nuevas alternativas de gobernanza para los problemas de sustentabilidad ambiental y social (plantaciones) y económica (bosque nativo) del sector forestal. La figura 1 muestra los principales hitos relativos al surgimiento y evolución de la certificación forestal en Chile.

Los primeros intentos para introducir un sistema de certificación de manejo forestalmente sustentable, a través del FSC, surgieron en la segunda mitad de la década de 1990. En particular, ciertos miembros de ONG nacionales entrevistados, veían al FSC como un método concreto para implementar los principios, criterios e indicadores de manejo forestal sustentable establecidos en el proceso de Montreal y acordados por varios países miembros en la "Declaración de Santiago" en Chile.

Sin embargo, la industria forestal no reaccionó sino hasta que una campaña internacional arriesgó su credibilidad y acceso a mercados internacionales. Esta campaña la lideró la ONG Forest Ethics: el 13 de septiembre de 2002 publicó un aviso de media página en el New York Times en el que mostraba una tala rasa y llamaba a los compradores estadounidenses a dejar de comprar productos forestales de empresas chilenas, a menos que se certificaran bajo el estándar FSC. La campaña atrajo la atención hacia el destino de miles de hectáreas de bosque nativo templado sustituidas por plantaciones y su impacto fue tan fuerte, que de acuerdo a ciertos investigadores forestales entrevistados, obligó a buscar un acuerdo. Este acuerdo, promovido por Home Depot se conoce como Joint Solutions Project (ver Heilmayr y Lambin 2016). De esta manera, en 2003, las más grandes empresas (Forestal Mininco S.A., Forestal Arauco S.A., y posteriormente MASISA S.A.) acordaron con Forest Ethics y otras ONG ambientales no substituir sus bosques nativos.

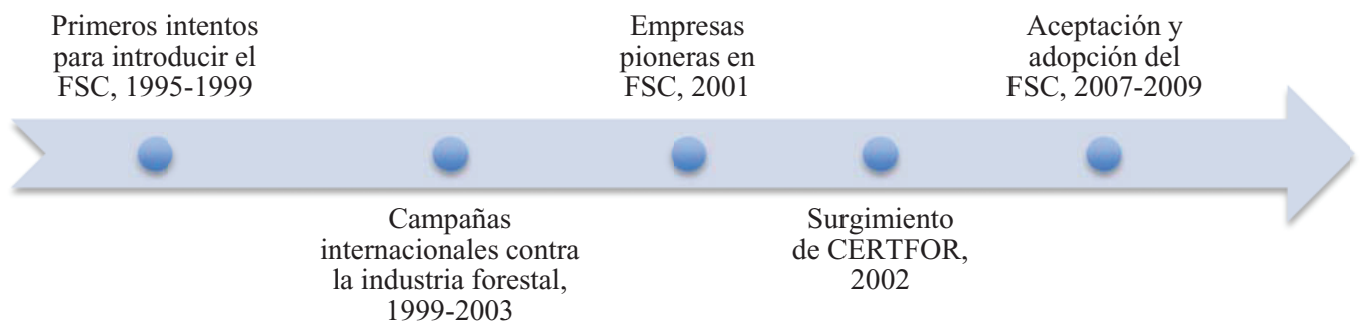

Figura 1. Principales eventos en el surgimiento y evolución de la certificación forestal en Chile.

Main milestones in the rise and evolution of forest certification in Chile. 
A partir del año 2001, dos empresas de plantaciones controladas por multinacionales, Forestal Millalemu y Forestal Monteáguila, fueron las primeras en obtener el sello FSC marcando un precedente para la industria forestal. No obstante, con apoyo financiero de agencias estatales, la mayor parte de la gran industria forestal prefirió crear y adoptar su propio estándar alternativo en 2002: CERTFOR. Según reconocieron fuentes de la gran industria forestal, CERTFOR surgió debido a que sintieron que el FSC les impondría un monopolio y requerimientos difíciles de cumplir.

Para la mayoría de los entrevistados, sin embargo, incluyendo ONG, consultores e investigadores forestales y propietarios de pequeñas plantaciones, CERTFOR carecería de legitimidad debido a que en la creación del estándar no estaban representados todos los actores. Hasta hoy, las principales ONG nacionales han continuado apoyando FSC sobre CERTFOR. Pese a lo anterior, CERTFOR fue reconocido internacionalmente bajo el alero de PEFC (Programme for the Endorsement of Forest Certification), $\mathrm{y}$ ha sido exitoso en términos de superficie certificada sumando más de 1,9 millones de hectáreas de plantaciones (CertforChile 2015).

Últimamente el FSC fue aceptado y adoptado en 2009 por la industria forestal que era inicialmente renuente a su adopción. De acuerdo a la mayoría de los entrevistados, hay ciertas razones que explicarían esto. Primero, la incorporación de representantes de la gran industria forestal en la cámara económica del FSC abrieron el camino a que la industria pudiera influir "desde adentro" en la gobernanza del estándar. Esto ha abierto un debate en ciertas ONG nacionales sobre la excesiva influencia de los intereses de las empresas en el FSC nacional. Segundo y tal vez más importante, la gran industria de plantaciones forestales fue presionada por algunos de sus clientes en el hemisferio norte de que no aceptarían CERTFOR como alternativa válida de manejo forestal sustentable: debían estar certificados en FSC. Hoy en día, más de 2,3 millones de hectáreas de plantaciones y bosque nativo están certificadas bajo el estándar FSC (FSC Chile 2015). Finalmente, cabe señalar que a pesar de haber existido apoyo inicial para crear CERTFOR y, según autoridades forestales entrevistadas, un acuerdo en 2013 entre FSC Chile y CONAF (para favorecer la adopción de $\mathrm{FSC}$ por pequeños propietarios), no existe un apoyo estatal sistemático bajo subsidios u otros instrumentos para promover la certificación forestal.

\section{DISCUSIÓN}

Los estudios de caso expuestos en este trabajo son, en general, consistentes con otros estudios acerca de la industria forestal chilena. En el caso del establecimiento de las plantaciones forestales, sus efectos negativos ambientales y sociales concuerdan con otros estudios (Gerber 2011, Nahuelhual et al. 2012, Meyer y Tappin 2014, Reyes y Nelson 2014, Andersson et al. 2016). Ambientalmente, muchas operaciones forestales no seguirían prácticas sos- tenibles, demostrando que los instrumentos de gobernanza tradicional no asegurarían el cumplimiento de las regulaciones forestales. Socialmente, si bien la gran industria forestal sería exitosa económicamente, comunidades locales, pequeñas empresas forestales, contratistas, trabajadores y grupos indígenas no habrían obtenido beneficios equitativos de este crecimiento. Más bien, los beneficios se concentrarían principalmente en los propietarios de estas grandes corporaciones. Además, se identificaron importantes asimetrías de poder entre grandes empresas forestales versus contratistas y comunidades locales; las cuales eran a su vez replicadas entre contratistas y trabajadores forestales, en desmedro del componente más débil en la ecuación.

No sorprende entonces, que las plantaciones forestales sean resistidas por ciertas ONG y comunidades locales, así como también que conflictos de alta intensidad con comunidades indígenas se hayan multiplicado en años recientes. De manera importante, aunque la mayor parte de los impactos ambientales y sociales de las grandes empresas estaría asociada a la mayor extensión e intensidad de sus operaciones forestales, no se podría asegurar esto en términos de impacto por hectárea, pues descontando los modelos agroforestales, existirían numerosas pequeñas y medianas empresas con prácticas forestales deficientes. Es necesario, sin embargo, más investigación para profundizar en esto último.

El sector bosque nativo analizado, descontando las numerosas pequeñas operaciones forestales ilegales, tendría menores impactos ambientales y sociales. Ambientalmente, esto se debería a que las operaciones forestales serían ejecutadas con métodos de baja intensidad. En materia social, los estudios de caso revelaron precarias condiciones de salud y seguridad ocupacional, consistente con otros autores (Otero 2006). Si bien la menor extensión de las operaciones subestimaría este impacto negativo, será necesaria más investigación en esta área.

Económicamente, este trabajo aporta nuevos antecedentes a las causas del modesto desempeño del sector bosque nativo, lo cual es consistente con el trabajo de Bustamante y Díaz (2010). Sumado a esta incierta sustentabilidad económica, las operaciones formalizadas, según varios entrevistados reconocieron, enfrentarían excesivas restricciones y burocracia estatal. Esto sería contraproducente pues estimularía a más propietarios a realizar sus operaciones de manera ilegal. Más aún, los intentos por dinamizar este sector mediante la ley de bosque nativo (ley 20283) no habrían dado los beneficios esperados.

En general, este trabajo no solo concuerda con estudios anteriores en el sector forestal plantaciones y bosque nativo, sino que expande sus resultados y provee nuevos hallazgos. Importantemente, la gobernanza estatal forestal basada en instrumentos de regulación convencional y económicos, incluyendo exenciones tributarias y subsidios, no ha sido ni estaría siendo lo suficientemente eficaz ni flexible ante los problemas del sector. Más aún, según varios entrevistados, las limitadas atribuciones y recursos de CONAF y la Dirección del Trabajo en fiscalizar el cumpli- 
miento legal de las empresas harían que muchas regulaciones no se cumplieran en la práctica.

En consecuencia, el surgimiento de la certificación de manejo forestal respondería principalmente a la presión de ONG nacionales e internacionales, así como de consumidores internacionales, para mejorar la sustentabilidad de la industria forestal chilena; actores quienes no percibieron suficientes garantías en la gobernanza estatal de inicios de este siglo. La mayoría de las empresas, por su parte, habría adoptado la certificación para obtener "licencia social para operar" desde estos actores, y mantener/ganar mercados; siendo el caso chileno similar a ejemplos en otros países (Auld 2014).

\section{CONCLUSIONES}

Dos importantes conclusiones se pueden obtener de este estudio. En primer lugar, la significativa brecha en sostenibilidad ambiental y social de las grandes plantaciones forestales no ha sido subsanada suficientemente por el progreso de regulaciones estatales tradicionales. Más bien, en el caso de conflictos con comunidades indígenas, algunas leyes (ley 19253 de 1993) los han agravado. Sería recomendable entonces, que desarrollos regulatorios nuevos o innovadores establecieran reglas claras y equitativas para todos los actores clave en gobernanza forestal.

En segundo lugar, de acuerdo a los estudios de caso analizados, el sector formal de bosque nativo y ciertos pequeños y medianos propietarios de plantaciones forestales tuvieron, relativamente, las menores brechas ambientales y sociales, pero una amplia brecha en términos económicos. Lo anterior tampoco ha sido subsanado por los instrumentos de política forestal estatal, ni en la forma de subsidios por manejo forestal sustentable ni a través de programas de "compras verdes" del estado. Consecuentemente, las recomendaciones de política pasan por generar incentivos económicos más eficaces o flexibles que los existentes hoy en día.

Como conclusión general, y respondiendo a la hipótesis de investigación planteada al inicio, los instrumentos de gobernanza tradicional no han respondido a los desafíos de sustentabilidad ambiental, social y económica del sector forestal chileno en su conjunto. No sorprende entonces que este contexto haya propiciado y propicie la adopción de la certificación forestal como un instrumento de gobernanza alternativo, no-estatal.

Sin embargo, más investigación es necesaria para evaluar la efectividad de la certificación forestal y para profundizar en temas menos investigados como lo es la sustentabilidad del sector de bosque nativo y pequeñas y medianas operaciones forestales en general.

\section{AGRADECIMIENTOS}

Se agradece a la Comisión Nacional de Ciencia y Tecnología (CONICYT) de Chile por el financiamiento otorgado para este trabajo. Asimismo, a los profesores Dr. Pe- ter Kanowski, Dr. Neil Gunningham y al Dr. Fred Gale por su invaluable supervisión. Finalmente, se agradecen las valiosas sugerencias del Dr. Felipe Meyer y de los árbitros de la revista Bosque.

\section{REFERENCIAS}

Andersson K, D Lawrence, J Zavaleta, MR Guariguata. 2016. More Trees, More Poverty? The Socioeconomic Effects of Tree Plantations in Chile, 2001-2011. Environmental Management 57(1): 123-136. DOI: 10.1007/s00267-0150594-x

Attride-Stirling J. 2001. Thematic networks: an analytical tool for qualitative research. Qualitative Research 1: 385-405. DOI: $10.1177 / 146879410100100307$

Auld G. 2014. Constructing Private Governance: The Rise and Evolution of Forest, Coffee, and Fisheries Certification. New Haven, United States. Yale University Press. 352 p.

Tricallotis M, P Kanowski. 2016. Background Paper: tree plantations in the landscape - Concepción/Temuco, Chile. The Forests Dialogue, Yale. Consultado 01 ago. 2016. Disponible en http://theforestsdialogue.org/dialogue/field-dialogue-tree-plantations-landscape-tpl-chile

Bustamante JA, JG Díaz. 2010. Tipificación de los sistemas de producción en productores de leña certificada, de las comunas de Mariquina, Corral y Valdivia, en la región de Los Ríos. Bosque Nativo 46:3-11.

CertforChile (Corporación CertforChile, CL). 2015. Empresas certificadas: Manejo Forestal Sustentable. Consultado 23 jul. 2015. Disponible en http://www.certfor.org/certificadas.php

Heilmayr R, EF Lambin. 2016. Impact of nonstate, market-driven governance on Chilean forests. PNAS 113(11): 29102915. DOI: $10.1073 /$ pnas. 1600394113

INFOR (Instituto Forestal, CL). 2016. Estadísticas Forestales. Consultado 15 ago. 2016. Disponible en http://wef.infor.cl/

De Groot RS, PJ van der Meer. 2010. Quantifying and valuing good and services provided by plantation forests. In Bauhus J, P van der Meer, M Kanninen eds. Ecosystem Goods and Services from Plantation Forests. UK, USA. Earthscan. p. 16-42.

FSC-Chile (Forest Stewardship Council, CL). 2015. Superficie Certificada FSC-Chile. Consultado 02 abril 2015. Disponible en https://cl.fsc.org/es-cl/certificacin/superficie-yempresas-cetificadas-en-chile

Gerber J. 2011. Conflicts over industrial tree plantations in the South: Who, how and why? Global Environmental Change 21:165-176.

Hopkin J. 2010. The Comparative Method. In Marsh D, G Stoker eds. Theory and methods in political science. Basingstoke, UK. Palgrave Macmillan. p. 285-307.

INFOR (Instituto Forestal, CL). 2015. Anuario Forestal 2015. Santiago, Chile. INFOR. 174 p. (Boletín Estadístico N ${ }^{\circ}$ 150).

Kausel T, C Vergara. 2003. El uso de la leña como combustible en le IX región. Aspectos económicos. In Burschel H, A Hernández, M Lobos eds. Leña - una fuente energética renovable para Chile. Santiago, Chile. Editorial Universitaria. p. 41-54.

Lara A, R Reyes, R Urrutia. 2013. Capítulo 3, Bosques Nativos. In Universidad de Chile, Instituto de Asuntos Públicos 
(INAP), Centro de Análisis de Políticas Públicas (CAPP). 2013. Informe País, Estado del Medio Ambiente en Chile. Santiago, Chile. p. 141-188.

Layder D. 1998. Sociological practice: linking theory and social research. London, UK. Sage Publications. 308 p.

Leyton JI. 2009. Tenencia Forestal en Chile. Organización de las Naciones Unidas para la Agricultura y la Alimentación (FAO) (Estudios de Caso). Consultado 23 jul. 2015. Disponible en http://www.fao.org/forestry/54367/es/chl/

Meyer F, D Tappin. 2014. Social Sustainability in the Chilean Logging Sector. In Sun W ed. Corporate Social Responsibility and Sustainability: Emerging Trends in Developing Economies. Emerald Group Publishing. p. 269-294. DOI: 10.1108/S2043-905920140000008014

Nahuelhual L, A Carmona, A Lara, C Echeverría, ME González. 2012. Land-cover change to forest plantations: proximate causes and implications for the landscape in south-central Chile. Landcsape and Urban Planning 107: 12-20.

Neuman WL. 2011. Social Research Methods: Qualitative and Quantitative Approaches. 7th ed. Upper Saddle River, US.
Pearson Education. 640 p.

Otero L. 2006. Precertificación forestal de comunidades indígenas en Chiloé y Osorno. In Catalán R, P Wilken, A Kandzior, D Tecklin, H Burschel eds. Bosques y Comunidades del Sur de Chile. Santiago, Chile. Editorial Universitaria. p. 199-209.

Reyes R, H Nelson. 2014. A tale of two forests: why forests and forest conflicts are both growing in Chile. International $\mathrm{Fo}$ restry Review 16:379-388.

Gunningham, N. 2009. The new collaborative environmental governance: The Localization of Regulation. Journal of Law and Society 36(1): 145-166. DOI: 10.1111/j.14676478.2009.00461.x

Tikina AV, JL Innes JL. 2008. A framework for assessing the effectiveness of forest certification. Canadian Journal of Forest Research 38: 1357-1365. DOI: 10.1139/X08-011

OIT Santiago. 2012. El trabajo decente en la Industria Forestal en Chile. Consultado 01 ago. 2016. Disponible en http:// www.ilo.org/santiago/publicaciones/WCMS_206093/lang-es/index.htm 